\title{
Direct Actions of Ovarian Hormones and the Related Compounds on the Uterine Muscle in vitro
}

\author{
By \\ Keisuke Okamura, Akinori Nishiyama and Taizo Suzuki \\ Department of Applied Physiology, Tohoku University, School of \\ Medicine, Sendai
}

(Received for publication, August 30, 1962)

\begin{abstract}
INTRODUCTION
Although there exists a considerable number of literatures on the regulatory actions of the ovarian hormones on the uterine muscle, little is known of their mode of action. It is, however, generally recognized that estrogen is an activating substance for the uterus; when estrogen is withdrawn (by ovariectomy), its spontaneous contraction and the maximum tension produced by an electrical stimulation are markedly decreased, but the repeated treatment with estrogen resulted in a gradual return to estrogen dominated active status. Progesterone, on the other hand, exhibits decrease of contractility and hyposensitivity of excitation, and this inactivation effect of progesterone is believed to play a cardinal role for the maintenance and termination of pregnancy. Recently, Corner and Csapo, ${ }^{1)}$ and Okamura ${ }^{2}$ demonstrated the specific action of progesterone on the uterus as indicated by the negative stair-case (Corner and Csapo) or the Transitional phenomenon (Okamura). These effects of ovarian hormones are produced by giving them directly to the experimental animals.

St. Györgyi, ${ }^{3)}$ on the other hand, described on the direct action of steroids that the positive stair-case seen in isolated frog heart was abolished by desoxycorticosterone (DOC) or progesterone when applied directly into the perfused solution. Csapo and Kuriyama ${ }^{4,5)}$ reported that when progesterone was directly applied on the estrogen dominated uterus in vitro, the maximum tension produced by electrical stimulation gradually decreased and the membrane potential was hyperpolarized in some degree. These effects were completely reversible upon washing with normal Krebs solution.

Then, the question arises whether response of the uterus to progesterone produced by giving hormone in vivo to the animal and its direct application in vitro are exactly the same or not. If not, does the ovarian hormone possess a
\end{abstract}

岡村 桂介, 西山 明德, 鈴木 彔三 
dual action of the direct and indirect effect on the uterus? Thirdly, is progesterone the only hormone which brings about the inactivation of uterine contractility?

The present paper reports the results of the direct action of ovarian hormones and the related steroids on the estrogen dominated rabbit uterus in vitro. An investigation was also made of the interactions among these steroids.

\section{METHODS}

White female rabbits weighing 1.5 to $2.5 \mathrm{~kg}$. were spayed and left alone for about 2 weeks before the hormonal treatment. They were then received i.m. injection of estradiol benzoate $25 \gamma / \mathrm{kg} /$ day for 7 days to obtain the estrogen dominated uteri. Uterus was removed by quick laparotomy without anesthesia and immediately immersed in Krebs solution of $10^{\circ} \mathrm{C}$, keeping unstimulated and care was taken to maintain the temperature of solution around $10^{\circ} \mathrm{C}$ and the material of a few hours after the removal was discarded, for the stock of uterine strip for a long time is thought to shift the membrane potential to depolarization.

The composition of Krebs solution used in these experiments was as follows $(\mathrm{mM}): \mathrm{NaCl} 138, \mathrm{KCl} 3.1, \mathrm{MgCl}_{2} 0.2, \mathrm{CaCl}_{2} 1.4$, phosphate buffer and glucose. $\mathrm{pH}$ was 7.3 .

A muscle strip was cut $2 \mathrm{~cm}$. long in resting length and mounted in a bath containing $100 \mathrm{ml}$. Krebs solution of $28^{\circ} \mathrm{C}$ with constant bubbling of oxygen. Following a 10 minutes period of rest, electrical stimulations were applied repeatedly to the muscle strip which gave isometric contraction.

Tension produced by contraction was led to mechano-electric transducer (RCA 5734), amplifier and finally to ink-writing oscillograph. Resting tension of every strip was not exceeded $1 \mathrm{~g}$.

Ovarian hormones and its related steroids used in this experiment were estradiol, testosterone, progesterone, DOC, cortisone, Viadril and 4-chlorotestosterone (anabolic steroid), which were added to Krebs solution rapidly in various amount and well mixed. These hormones or steroids were given when the maximal contraction height was reached and the influence was observed for 30 minutes. Then, the muscle strip was washed with normal Krebs solution for several times and recovery process was traced for additional 30 minutes.

Electrical stimulation was applied longitudinally to the strip, which voltage was $2 \mathrm{~V} / \mathrm{cm}(50 \mathrm{c} / \mathrm{s}$ a.c.) and is considered to be optimal to produce the maximum tension. Duration and interval of the repeated stimuli were 5 and 40 seconds, respectively. To examine the influence of these hormones or steroids to the contractile system of uterine muscle, $10 \mathrm{~V} / \mathrm{cm}$ of stimulus was given once in a series of $2 \mathrm{~V} / \mathrm{cm}$ stimuli. 


\section{RESULTS}

\section{Dose response}

Various kinds of steroids were examined in this experiment, in which five of them showed inhibitory action for the uterine contraction after the application of a steroid directly into Krebs solution. Fig. 1 indicates the rate of inhibition of contraction by steroids. Testosterone, progesterone and cortisone exhibited apparent inhibitory action in small amount and the rate of inhibition was parallel to the dose given except cortisone, which gave no significant change in double dose. The effect of DOC was very mild even in large dose. Viadril, on the contrary, showed marked inhibition especially in large amount. Estradiol demonstrated no direct action of inhibition in any dose. 4-chlorotestosterone also exhibited no depressive action to the uterine contraction even in a huge dose of 20 mg.
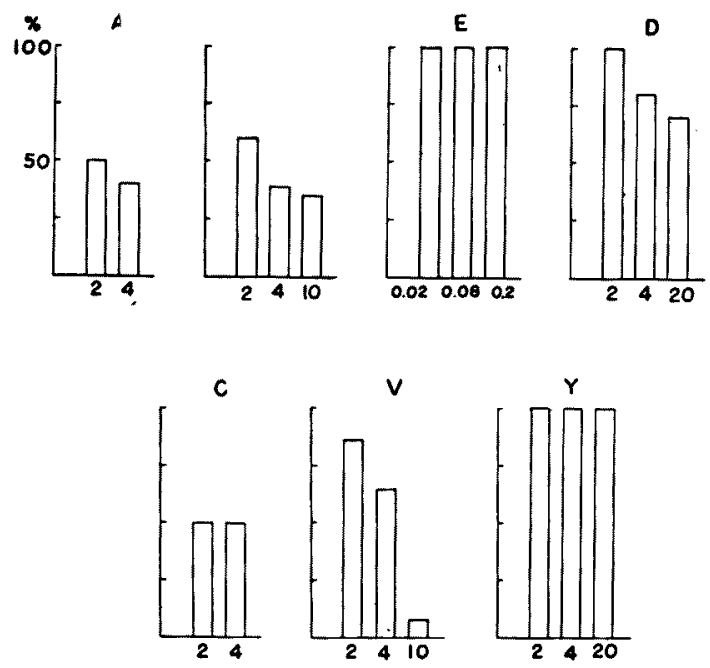

Fig. 1. Dose response of various steroids to the uterine muscle. Ordinate shows the rate of the maximal contraction height in \%. Abscissa indicates the dose of steroid in $\mathrm{mg} / 100 \mathrm{ml}$. A, testosterone; $\mathrm{P}$, progesterone; E, estradiol; $\mathrm{D}, \mathrm{DOC} ; \mathrm{C}$, cortisone; $\mathrm{V}$, Viadril; $\mathrm{Y}, 4$-chlorotestosterone. In $\mathrm{D}$, about a half of cases showed no inhibiting action even $4 \mathrm{mg}$ of dose.

\section{Recovery}

Is the inhibitory action of the above mentioned steroids reversible? For this investigation, 30 minutes were allowed, following the hormonal treatment, for the uterine strip after several rinses with pure Krebs solution. In the present experiment, the applied dose of a steroid was $2 \mathrm{mg}$. in every case. 
Progesterone, estradiol, DOC, Viadril and 4-chlorotestosterone revealed their scovery to be complete accompanied by no sequent influence. But further ıvestigations with increased dose of steroids revealed the recoveries of proge;erone and DOC were unsatisfactory, though estradiol still kept good recovery. 'estosterone and cortisone seemed to produce some irreversible influence on the terine muscle.

\section{Time course for the response}

After appling $4 \mathrm{mg}$. of a steroid in a single dose, an observation was made for 0 minutes to follow the response of uterine muscle. As seen in Fig. 2, progesterne and testosterone demonstrated typical diminishing effect in contraction of terine muscle to the electrical stimulation. While in progesterone, a linear ecrease of tension was observed, testosterone and cortisone exhibited an xponential diminution or rather abrupt depression of tension within 5 minutes. The inhibiting action of cortisone and Viadril was not so remarkable as progeterone and testosterone. Estradiol, 4-chlorotestosterone and DOC* were found o be not effective.

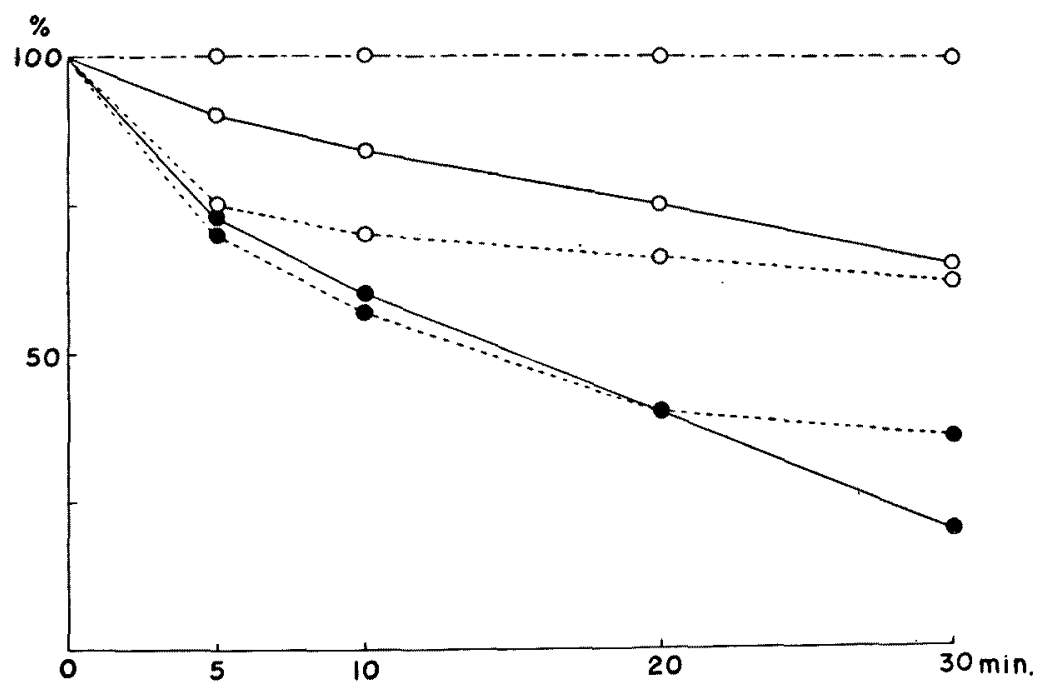

Fig. 2. Time course for the response to various steroids. Ordinate, rate of the maximal contraction height in \%. Abscissa; time in minute. $\bullet-, P$; $\cdots \bullet, A ; 0 \cdots 0$ C; $\circ, \mathrm{V} ; 0 \cdots 0, E, D, Y . A, P, E, D, C, V$ and $Y$, refer to Fig. 1.

\section{Interaction between two steroids}

It is of significant to examine the interaction between two kinds of steroids

* Some of the cases showed a mild depressing effect. 

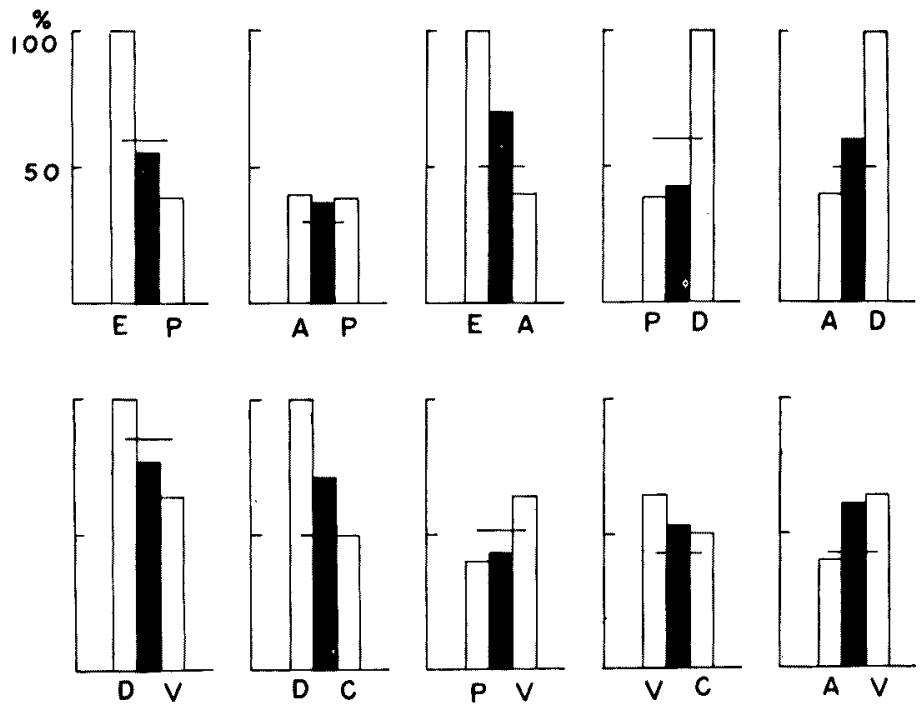

Fig. 3. Interaction between two steroids. Ordinate: rate of the maximal contraction height in \%. Open column: $4 \mathrm{mg}$ of single steroid, except $\mathrm{E}$ (which is $0.02 \mathrm{mg}$ ). Shaded column: $2 \mathrm{mg}$ of two steroids given togather. A,P,E,C,D,V and $\mathrm{Y}$ : refer to Fig.

1. A bar in each graph represents its expected value.

when applied at the same time to the uterine muscle. Another word, one of the actions of synergism, antagonism or simple summing-up between two steroids should be expected (Fig. 3).

Assuming their interaction to be a simple mathematical summing-up, the expected values of each combination were obtained (refer to Fig. 1). When the expected and the obtained values were compared in a graph, the interaction between two steroids became apparent; in which a supposition that the inhibitory action of steroids to the uterine muscle contraction was independent each other, turned out to be incorrect. Namely, none of the combinations showed the depressing effect in contraction by a simple summing-up of two steroids.

The antagonistic effect between two steroids was obtained in the following combinations; testosterone + progesterone $(-7)$, testosterone + estradiol $(-20)$, testosterone +DCC $(-10)$, testosterone + Viadril $(-18)$, cortisone + Viadril $(-10)$, cortisone +DOC $(-21)$; a number in parenthesis means the difference between the obtained value and the expected one, expressed in per cent of the initial contraction height. Testosterone seemed to have an antagonistic activity to any other steroids combined, resulting in less inhibitory action of uterine contraction than their expected value.

Synergetic action was observed in progesterone + estradiol $(+4)$, progesterone + DOC $(+17), \mathrm{DOC}+$ Viadril $(+9)$ and progesterone + Viadril $(+9)$; their combinations potentiated the inhibitory action of uterine contraction. 
Response to strong stimulus

Csapo $\left.^{6}\right)$ reported that when estrogen dominated uterus is stimulated with icreasing strength of electrical stimulus step by step, tension first increases, then ecreases and again increases and decreases, to leave two peaks behind on the zcord. These two peaks appear to about $2 \mathrm{~V} / \mathrm{cm}$ and about $10 \mathrm{~V} / \mathrm{cm}$, respectively. t $2 \mathrm{~V} / \mathrm{cm}$, muscle is stimulated through the normal excitation, but at $10 \mathrm{~V} / \mathrm{cm}$, he myoplasm or coupling mechanism is thought to be stimulated directly.

Testing the response to strong stimulus $(10 \mathrm{~V} / \mathrm{cm})$, it became evident that he inhibitory action of progesterone was but slight, if at all effective. 'estosterone and cortisone, on the contrary, demonstrated the diminishing ffect in contraction to strong stimulus (Fig. 4). These results mean chat under he effect of progesterone, excitability was reduced but contractility was almost naltered, whereas testosterone and cortisone impaired both excitability and ontractility.

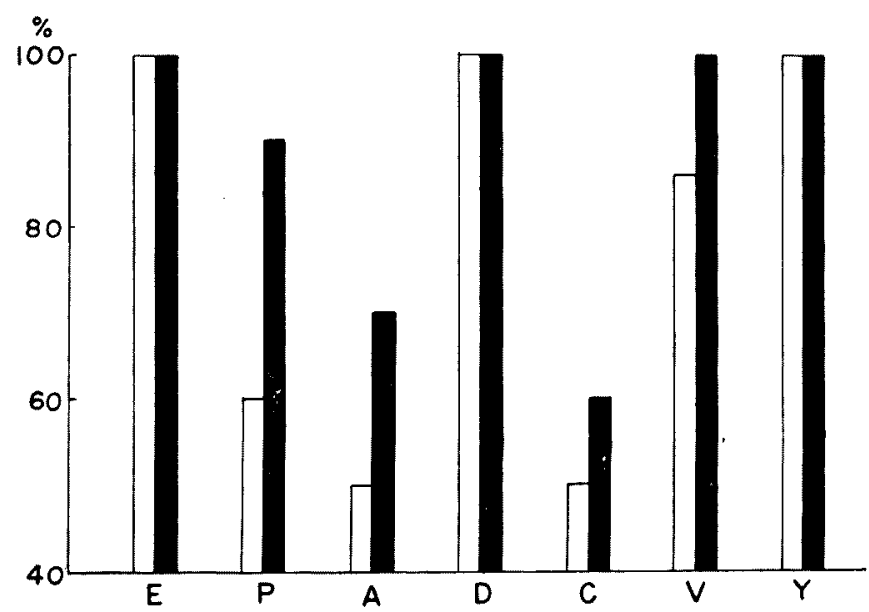

Fig. 4. Response to electrical stimulus of $10 \mathrm{~V} / \mathrm{cm}$ under the treatment of steroid $(2 \mathrm{mg})$. Ordinate: rate of the maximal contraction height in \%. Open column: response to $2 \mathrm{~V} / \mathrm{cm}$. Shaded column: response to $10 \mathrm{~V} / \mathrm{cm}$.

\section{DISCUSSION}

Since Corner's classical observation, it has been widely accepted that progesterone exhibits the inhibitory action to the uterine motility, when it is given in vivo. But, Csapo and Kuriyama ${ }^{4,5)}$ have recently described that progesterone produced the diminution of contraction height of uterus, even it is given in vitro. The mechanism of the direct action of progesterone on the uterine motility is still not exactly understood. Kuriyama and Csapo," Marshall and Csapo ${ }^{8)}$ reported 
that the hyperpolarization in membrane potential in muscle cell was induced by the treatment of progesterone in vivo and also in vitro. So far as progesterone is concerned, it is accepted from the present experiment that the inhibitory action of steroid is due to the decreased excitability of the membrane. Another word, under the direct effect of progesterone, excitability was profoundly changed, whereas the contractility was left almost unchanged. There are, however, some complications. Testosterone and cortisone seemed to attack deeper system by the fact that these steroids revealed an apparent effect of inhibition produced by stimuli of $2 \mathrm{~V} / \mathrm{cm}$ and $10 \mathrm{~V} / \mathrm{cm}$. As Csapo ${ }^{4}$ pointed out, progesteronelike compounds or progesterone metabolites (pregnanediol, pregnanedione, pregnanolone), which have no effect on the maintenance of pregnancy, are effective in vitro, whereas, others (for example, 17-hydroxyprogesterone, corticosterone) are effective in vivo, but ineffective in vitro. The cause of the difference between direct and indirect actions on uterine muscle is obscure at present and further investigation should be performed.

Concerning to the interaction of steroids, numerous clinical reports have been made to improve on steroid therapy. But, little is known on the effect of combination treatment of two kinds of steroid in vitro upon the contractility of uterine muscle. The present experiment on the interaction of two steroids demonstrated the synergetic and antagonistic pattern of activities on the uterine contraction. A finding by Stucki et al. ${ }^{9)}$ on the inhibition of parturition in vivo agreed with the present data that progesterone and testosterone block the uterine motility. The mechanism by which additive-inhibitory activities of two steroids are brought about are not known at present and further study is indicated.

\section{SUMMARY}

Investigations were made on the direct action of various steroid hormones and the related compounds in contraction produced by electrical stimulation. Estrogen dominated rabbit uterus was used for experiment.

1. Progesterone, cortisone and testosterone showed an apparent diminution of uterine contraction, while the inhibitory action of Viadril and DOC was of a very mild degree in small dose.

2. In a dose of $2 \mathrm{mg}$, progesterone, estradiol, DOC, Viadril and 4chlorotestosterone revealed to be complete, but testosterone and cortisone produced some irreversible influence on the uterine muscle.

3. Progesterone showed a linear decrease of tension when the time elapsed. Testosterone and cortisone exhibited an exponential diminution of contraction height.

4. On the interaction of steroids, while antagonistic effect was observed in the combination of testosterone+progesterone, testosterone+estradiol, 
iestosterone $+\mathrm{DOC}$, testosterone + Viadril, cortisone + Viadril and cortisone $+\mathrm{DOC}$, synergetic action was obtained in progesterone +estradiol, progesterone+DOC, $\mathrm{DOC}+$ Viadril and progesterone+Viadril.

5. To strong stimulus, when testosterone and cortisone kept a marked nhibitory action, progesterone gave almost no change in contraction.

\section{Acknowledgement}

The authors wish to express their thanks to Sanzen Pharmaceutical Prolucts and Yamanouchi Pharmaceutical Co. for 4-chlorotestosterone (Yonchlon), lesoxycorticosterone (Cortigen), progesterone (Progenin) and testosterone (Andogen), generously supplied for this study.

This work was supported by the Grant from the Population Council to one ff the authors (T. Suzuki).

\section{References}

1) Corner, G.W. \& Csapo, A., Brit. Med. J., 1953, 1, 687.

2) Okamura, K., Tohoku J. Exper. Med., 1962, 78, 32.

3) Szent-Györgyi, A., Chemical physiology of contraction in body and heart muscle, Academic press, 1953.

4) Csapo, A., Mechanism of action of steroid hormones, edited by C.A. Villee \& L.L. Engel, Pergamon press, 1961, p. 126.

5) Kuriyama. H., J. Physiol., 1961, 159, 26.

6) Csapo, A., Recent Progress in Hormone Research, 1956, 12, 405.

7) Kuriyama, H. \& Csapo, A., Endocrinol., 1961, 68, 1010.

8) Marshall, J.M. \& Csapo, A., Endocrinol., 1961, 68, 1026.

9) Stucki, J.G. \& Forbes, A.D., Acta Endocrinol., 1960, 33, 73. 